\title{
Radiofrequency ablation: A novel and effective treatment of severe bleeding secondary to actinic proctitis
}

\author{
Pilar Díez-Redondo, Carlos de-la-Serna, Ma Antonia Vallecillo, Henar Núñez, Paula Gil-Simón
} and Manual Pérez-Miranda

Department of Gastroenterology. University Hospital “Río Hortega”. Valladolid, Spain

\section{INTRODUCTION}

Some 5-20\% of radiotherapy treatments for pelvic tumours may cause a vascular rectal injury. Endoscopic treatments, fundamentally argon plasma coagulation (APC), are the most effective, but there are refractory cases and complications (1).

\section{CASE REPORT}

Male, 75-years-old. Cirrhosis and secondary thrombocytopenia. Eight months after radiation therapy for prostate adenocarcinoma, he presented actinic proctitis (AP) with severe rectal bleeding (haemoglobin $6.6 \mathrm{~g} / \mathrm{dL}$, platelets $28.000 / \mathrm{mm}^{3}$ ). After five sessions of APC and sucralfato, haemostasis was not achieved producing iatrogenic rectal ulcers (Fig. 1). He received 15 red blood cell packs and 2 platelets pools.

Ablation of the rectal mucosa with radiofrequency, Halo $60^{\circ}$ system, was proposed. Using a gastroscope at whose end was inserted the radiofrequency device, and with patient under propofol sedation, we applied power of $12 \mathrm{~J} / \mathrm{cm}^{2}$ and a density of $40 \mathrm{w}$, twice per area, with direct and retroverted view (Figs. 2 and 3). Only half the circumference was treated, to avoid stenosis, and also avoiding anorectal line. The patient's tolerance was very good. After the first session control of bleeding was achieved. A second session was scheduled at 4 weeks, but because of an intercurrent neurological process, was delayed at 7 weeks, and untreated mucosa ablation was performed. The third session, four weeks later, addressed residual

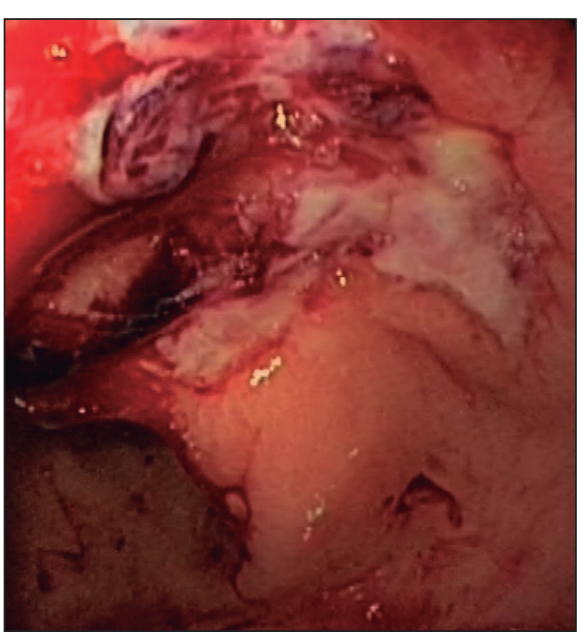

Fig. 1. latrogenic rectal ulcers post argon plasma coagulation treatment.

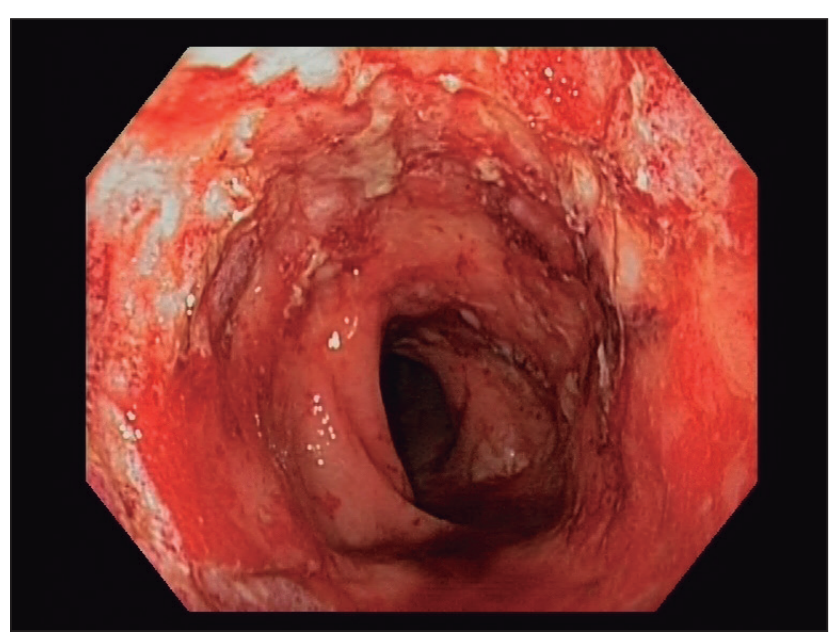

Fig. 2. After application of radiofrequency the mucosa exhibited a characteristic whitish colour that helps prevent overlapping by performing the following radiofrequency pulses. 
vascular lesions. At 10 weeks we verified the disappearance of neovessels and extensive reepithelialization (Fig. 4) that, in the area closest to the anal margin, was similar to endoanal mucosa and without complications. The patient has remained asymptomatic 8 months later.

\section{DISCUSSION}

The radiofrequency is used in the context of Barrett's esophagus, and also its usefulness has been described in some cases of antral vascular ectasia and AP (2-4).

We report a refractory AP bleeding in which mucosa ablation achieved maintained haemostasis. Destruction of most vascular lesions was achieved, yielding good reepithelialisation without complications.

Twice $12 \mathrm{~J} / \mathrm{cm}^{2}$ per area has demonstrated no involvement of the colonic serosa limiting involvement of the muscularis propia (5), and probably the low penetration make the ulcers or stenosis less likely than with other techniques.

Only three published articles have reported the usefulness of radiofrequency, using the Halo $90^{\circ}$, in this clinical entity (2-4). Using a small device, the Halo $60^{\circ}$, provides better manoeuvrability and a good visibility, facilitating getting as close as possible to the anorectal line without damaging it and it allows to treat wider areas than APC. Although because its cost is higher, should be reserved for cases refractory to conventional endoscopic treatment.

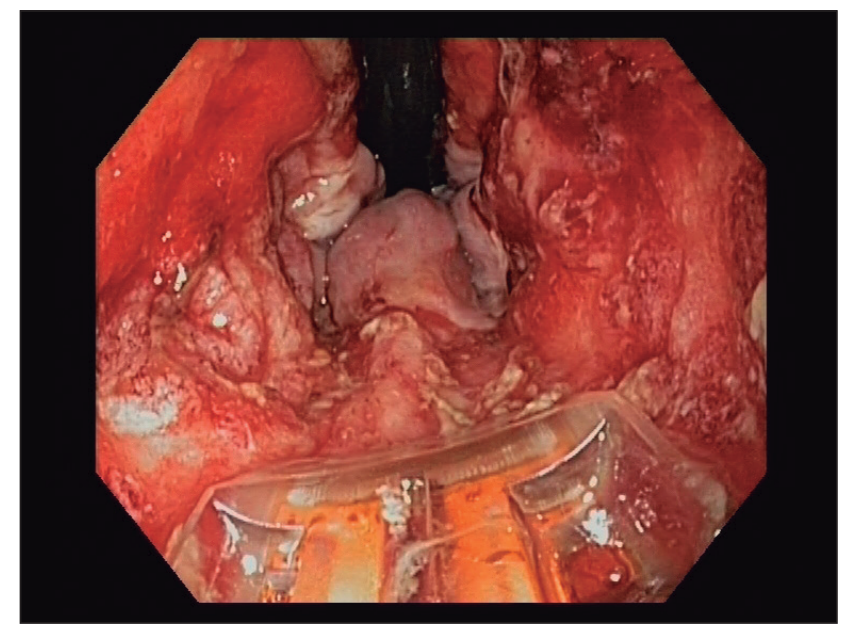

Fig. 3. Retroflex maneuver helps to application of radiofrequency energy with better contact of the device with the mucosa, and best visualization of the dentate line to avoid her damage.

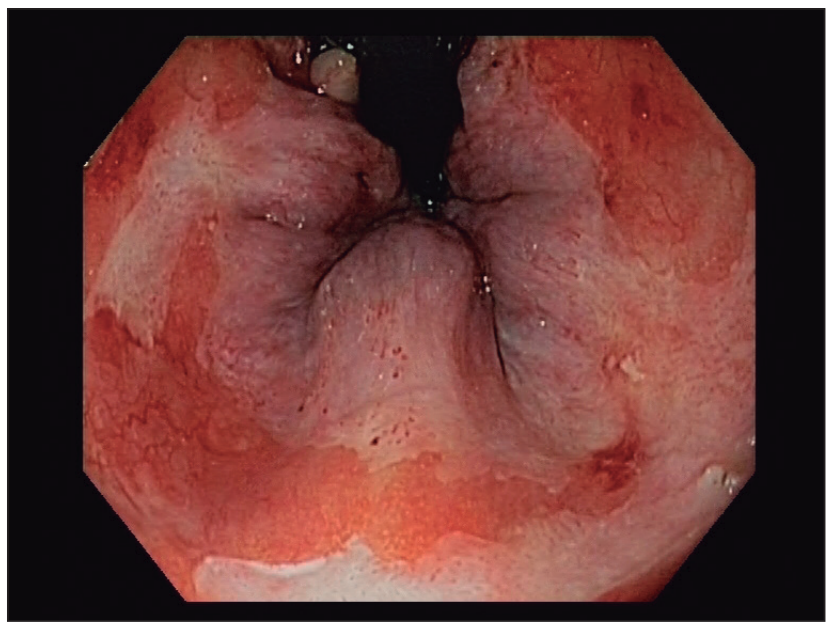

Fig. 4. Ten weeks after the last radiofrequency session we verified the disappearance of neovessels and extensive reepithelialisation, similar to anal mucosa in the area closest to the anal margin.

\section{REFERENCES}

1. Rustagi T, Mashimo H. Endoscopic management of chronic radiation proctitis. World J Gastroenterol 2011;41:4554-62

2. Zhou C, Adler D, Becker L, Chen Y, Tsai T, Figueiredo M. Effective treatment of chronic radiation proctitis using radiofrequency ablation. Therap Adv Gastroenterol 2009;2:149-56.

3. Nikfarjam M, Faulx A, Laughinghouse M, Marks JM. Feasibility of radiofrequency ablation for the treatment of chronic radiation proctitis. Surg Innov 2010;17:92-4.

4. Eddi R, DePasquale JR. Radiofrequency ablation for the treatment of radiation proctitis: A case report and review of literature. Therap Adv Gastroenterol 2013;6:69-76.

5. Trunzo JA, McGeeMF, Poulose BK, Willis JE, Ermlich B, Laughinghouse M, et al. A feasibility and dosimetric evaluation of endoscopic radiofrequency ablation for human colonic and rectal epithelium in a treat and resect trial. Surg Endosc 2011;25:491-6. 